\title{
Some New Existence and Uniqueness Results for an Integral Boundary Value Problem of Caputo Fractional Differential Equations
}

\author{
Haixing Feng ${ }^{1}$ and Chengbo Zhai $^{2}$ \\ ${ }^{1}$ College of Applied Mathematics, Shanxi University of Finance and Economics, Taiyuan 030031, China \\ ${ }^{2}$ School of Mathematical Sciences, Shanxi University, Taiyuan, Shanxi 030006, China \\ Correspondence should be addressed to Chengbo Zhai; cbzhai@sxu.edu.cn
}

Received 26 February 2017; Accepted 20 April 2017; Published 9 May 2017

Academic Editor: Chris Goodrich

Copyright (C) 2017 Haixing Feng and Chengbo Zhai. This is an open access article distributed under the Creative Commons Attribution License, which permits unrestricted use, distribution, and reproduction in any medium, provided the original work is properly cited.

\begin{abstract}
In this work, we consider an integral boundary value problem of Caputo fractional differential equations. Based on a fixed-point theorem of generalized concave operators, we obtain the existence and uniqueness of positive solutions. As applications of main results, we give two examples in the end.
\end{abstract}

\section{Introduction}

The fractional differential equations appear naturally in fields of physics, chemistry, electrodynamics of complex medium, control of dynamical systems, and so on. So differential equations with fractional order have been studied by many researchers, and then the theory of fractional boundary value problems has been noticed more and more in recent years; see [1-26], for example.

In [25], the author investigated the existence of positive solutions for an integral boundary value problem of fractional differential equations by using a fixed-point theorem on cones. In [21, 22], Yang gave the existence and nonexistence results for fractional differential equation integral boundary value problems. In these papers, we can find that there are no uniqueness results on positive solutions. Moreover, there are still very few results on the uniqueness of positive solutions for an integral boundary value problem of Caputo fractional differential equations. To fill this gap, we study the existence and uniqueness of positive solutions for the following integral boundary value problem for fractional differential equations

$$
\begin{aligned}
{ }^{C} D_{0^{+}}^{\alpha} x(t)+f(t, x(t)) & =0, \quad t \in(0,1), \\
a x(0)-b x^{\prime}(0) & =0
\end{aligned}
$$

$$
\begin{aligned}
c x(1)+d x^{\prime}(1) & =0 \\
x^{\prime \prime}(0)+x^{\prime \prime \prime}(0) & =\int_{0}^{1} x^{\prime \prime}(\tau) d p(\tau), \\
x^{\prime \prime}(1)+x^{\prime \prime \prime}(1)+\int_{0}^{1} x^{\prime \prime}(\tau) d q(\tau) & =0
\end{aligned}
$$

where $3<\alpha \leq 4, a, b, c, d \geq 0, \rho=a d+a c+b c>0$.

In [19], the authors studied the existence of positive solutions for (1) by the fixed-point theorems on cones. We also do not find the uniqueness results on positive solutions in [19]. In this paper, we will show the existence and uniqueness of positive solutions for problem (1). Our mail tool is a fixedpoint theorem of generalized concave operators in ordered Banach spaces.

The paper is organized as follows: in Section 2, we will present some useful definitions, preliminaries, and lemmas. The existence and uniqueness results are proved in Section 3. In Section 4, we finish the paper with two examples.

\section{Preliminaries}

Now we list some conditions for convenience. 
$\left(A_{1}\right) f(t, x) \geq 0, f(t, x)$ is increasing in $x \in[0,+\infty)$ for each $t \in[0,1]$.

$\left(A_{2}\right)$ For $\lambda \in(0,1)$, there exists $\alpha(\lambda) \in(\lambda, 1)$ such that $f(t, \lambda x) \geq \alpha(\lambda) f(t, x), t \in[0,1], x \geq 0$.

$\left(A_{3}\right) p(t), q(t)$ are right continuous on $[0,1)$, left continuous at $t=0$, and increasing on $[0,1]$, with $p(0)=q(0)=0 ; \int_{0}^{1} x(\tau) d p(\tau), \int_{0}^{1} x(\tau) d q(\tau)$ are the Riemann-Stieltjes integrals of $x$ with respect to $p, q$; moreover,

$$
\begin{aligned}
& 0 \leq \int_{0}^{1} d p(\tau)<1, \\
& 0 \leq \int_{0}^{1} d q(\tau)<1, \\
& \frac{2+\int_{0}^{1} \tau d q(\tau)}{1+\int_{0}^{1} d q(\tau)}>\frac{1-\int_{0}^{1} \tau d p(\tau)}{1-\int_{0}^{1} d p(\tau)} .
\end{aligned}
$$

$\left(A_{4}\right) f(t, x)$ is continuous with $\int_{0}^{1}(1-s)^{\alpha-4} f(s, x(s)) d s$ existing.

$\left(A_{1}^{\prime}\right) f(t, x)$ is decreasing in $x \in[0,+\infty)$ for each $t \in[0,1]$ and $f(t, x) \geq 0$ for $[0,1] \times[0,+\infty)$.

$\left(A_{2}^{\prime}\right)$ For any $\lambda \in(0,1)$, there exists $\beta(\lambda) \in(0,1)$ such that

$$
f(t, \lambda x) \leq \lambda^{-\beta(\lambda)} f(t, x), \quad t \in[0,1], x \geq 0 .
$$

In the following, we present some definitions and lemmas.

Definition 1 (see [12]). The Riemann-Liouville fractional integral of order $\alpha$ for function $y$ is

$$
I_{0^{+}}^{\alpha} y(t)=\frac{1}{\Gamma(\alpha)} \int_{0}^{t}(t-\alpha)^{\alpha-1} y(s) d s, \quad \alpha>0,
$$

provided that the right side is point-wise defined on $(0,+\infty)$.

Definition 2 (see [12]). The Riemann-Liouville fractional derivative of order $\alpha>0$ for function $y$ is

$$
D_{0^{+}}^{\alpha} y(t)=\frac{1}{\Gamma(n-\alpha)}\left(\frac{d}{d t}\right)^{n} \int_{0}^{t} \frac{y(s)}{(t-s)^{\alpha-n+1}} d s,
$$

$$
\alpha>0
$$

where $n=[\alpha]+1$, provided that the right side is point-wise defined on $(0,+\infty)$.

Definition 3 (see [23]). If $y \in A C^{n}[0,1]$, then the Caputo fractional derivatives of order $\alpha>0$ exist almost everywhere on $[0,1]$ and are represented by

$$
\begin{aligned}
{ }^{C} D_{0^{+}}^{\alpha} y(t) & =\frac{1}{\Gamma(n-\alpha)} \int_{0}^{t} \frac{y^{(n)}(s)}{(t-s)^{\alpha-n+1}} d s \\
& =\left(I_{0^{+}}^{n-\alpha} D^{n} y\right)(t) .
\end{aligned}
$$

Next we recall some concepts. Let $E$ be a real Banach space with norm $\|\cdot\|$, and let $\theta$ be the zero element of $E$. $P$ is a cone in $E$. For all $x, y \in E$, the notation $x \sim y$ means that there exist $\lambda>0, \mu>0$ such that $\lambda x \leq y \leq \mu x$. Clearly, $\sim$ is an equivalence relation. Given $h>\theta$, we denote by $P_{h}$ the set $P_{h}:=\{x \in E \mid x \sim y\}$. Clearly, $P_{h} \subset P$ is convex and $\lambda P_{h}=P_{h}$ for all $\lambda>0$.

Definition 4. A cone $P \in E$ is said to be normal if and only if there exists a constant $N>0$ such that

$$
\begin{gathered}
\theta \leq x \leq y \Longrightarrow \\
\|x\| \leq N\|y\|,
\end{gathered}
$$

$$
x, y \in E
$$

where $N$ is called the normality constant of $P$.

Definition 5. An operator $A: E \rightarrow E$ is increasing (decreasing) if $x \leq y$ implies $A x \leq A y(A x \geq A y)$.

Lemma 6 (see [19]). Suppose that $\left(A_{3}\right),\left(A_{4}\right)$ hold and $3<$ $\alpha \leq 4$; then the integral boundary value problem

$$
\begin{aligned}
{ }^{C} D_{0^{+}}^{\alpha} x(t)+f(t, x(t)) & =0, \quad t \in(0,1), \\
a x(0)-b x^{\prime}(0) & =0, \\
c x(1)+d x^{\prime}(1) & =0, \\
x^{\prime \prime}(0)+x^{\prime \prime \prime}(0) & =\int_{0}^{1} x^{\prime \prime}(\tau) d p(\tau), \\
x^{\prime \prime}(1)+x^{\prime \prime \prime}(1)+\int_{0}^{1} x^{\prime \prime}(\tau) d q(\tau) & =0
\end{aligned}
$$

has a solution

$$
x(t)=\int_{0}^{1} G_{1}(t, \xi) \int_{0}^{1} G_{2}(\xi, s) f(s, x(s)) d s d \xi
$$

where

$$
\begin{aligned}
& G_{1}(t, s)= \begin{cases}\frac{1}{\rho}(a s+b)[c(1-t)+d], & 0 \leq s \leq t \leq 1, \\
\frac{1}{\rho}(a t+b)[c(1-s)+d], & 0 \leq t \leq s \leq 1,\end{cases} \\
& G_{2}(t, s)=G_{\alpha}(t, s)+G_{p q}(t, s), \quad t, s \in[0,1], \\
& G_{\alpha}(t, s) \\
& \quad= \begin{cases}\frac{(1-s)^{\alpha-3}(1-t)+(t-s)^{\alpha-3}}{\Gamma(\alpha-2)}+\frac{(1-s)^{\alpha-4}(1-t)}{\Gamma(\alpha-3)}, & s \leq t, \\
\frac{(1-s)^{\alpha-3}(1-t)}{\Gamma(\alpha-2)}+\frac{(1-s)^{\alpha-4}(1-t)}{\Gamma(\alpha-3)}, & t \leq s,\end{cases}
\end{aligned}
$$




$$
\begin{aligned}
& G_{p q}(t, s)=\frac{1}{\delta}\left[\left(2+\int_{0}^{1} t d q(t)\right)-t\left(1+\int_{0}^{1} d q(\tau)\right)\right] \\
& \cdot \int_{0}^{1} G_{\alpha}(\tau, s) d p(\tau) \\
& +\frac{1}{\delta}\left[\left(1-\int_{0}^{1} t d p(t)\right)-t\left(1-\int_{0}^{1} d p(\tau)\right)\right] \\
& \cdot \int_{0}^{1} G_{\alpha}(\tau, s) d q(\tau), \quad t, s \in[0,1] \\
& \delta=\left|\begin{array}{ll}
1-\int_{0}^{1} d p(t) & 1-\int_{0}^{1} t d p(t) \\
1+\int_{0}^{1} d q(t) & 2+\int_{0}^{1} t d q(t)
\end{array}\right|>0 .
\end{aligned}
$$

Lemma 7 (see [19]). Let $3<\alpha \leq 4$. Then Green's function $G_{2}(t, s)$ has the following properties:

(i)

$$
\begin{aligned}
& G_{2}(t, s) \in C([0,1] \times[0,1]), \\
& G_{2}(t, s)>0
\end{aligned}
$$

(ii)

$$
t, s \in(0,1) \text {. }
$$

$$
\begin{aligned}
G_{2}(t, s) \geq m(t) M_{p q} M_{\alpha}(s), & \\
\max _{0 \leq t \leq 1} G_{2}(t, s) \leq M_{p q} M_{\alpha}(s), & \\
& t \in[0,1], s \in(0,1),
\end{aligned}
$$

where

$$
\begin{aligned}
& M_{p q}=1+\frac{1}{\delta}\left[\left(2+\int_{0}^{1} \tau d q(\tau)\right) \int_{0}^{1} d p(\tau)\right. \\
&+\left.\int_{0}^{1} d q(\tau)\right], \\
& m(t)=\frac{1}{M_{p q}}\left(\frac{1-t}{2}\right. \\
&+ \frac{1}{\delta}\left[\left(2+\int_{0}^{1} \tau d q(\tau)\right)-t\left(1+\int_{0}^{1} d q(\tau)\right)\right] \\
& \cdot \int_{0}^{1} \frac{(1-\tau)}{2} d p(\tau) \\
&+ \frac{1}{\delta}\left[\left(1-\int_{0}^{1} \tau d p(\tau)\right)-t\left(1-\int_{0}^{1} d p(\tau)\right)\right] \\
&\left.\cdot \int_{0}^{1} \frac{(1-\tau)}{2} d q(\tau)\right), t \in[0,1], \\
& M_{\alpha}(s)=\frac{2(1-s)^{\alpha-3}}{\Gamma(\alpha-2)}+\frac{(1-s)^{\alpha-4}}{\Gamma(\alpha-3)} .
\end{aligned}
$$

Lemma 8. Let $g(t)=\int_{0}^{1} G_{1}(t, s) d s$; then

$$
\begin{aligned}
& g(t)=\frac{1}{\rho}\left[-\frac{t^{2}}{2}(b c+a c+a d)+a\left(\frac{c}{2}+d\right) t\right. \\
& \left.+b\left(\frac{c}{2}+d\right)\right], \quad t \in[0,1]
\end{aligned}
$$

and $g(t) \geq g_{0}, t \in[0,1]$, where $g_{0}=\min \{(b / \rho)(c / 2+$ $d),(d / \rho)(a / 2+b)\}$.

We now present a fixed-point theorem of generalized concave operators which will be used in the later proofs.

Theorem 9 (see [27]). Let $h>\theta$ and $P$ be a normal cone. Assume that

$\left(D_{1}\right) A: P \rightarrow P$ is increasing and $A h \in P_{h}$;

$\left(D_{2}\right)$ for any $x \in P$ and $t \in(0,1)$, there exists $\alpha(t) \in(t, 1)$ with respect to $t$ such that

$$
A(t x) \geq \alpha(t) A x .
$$

Then

(i) there are $u_{0}, v_{0} \in P_{h}$ and $r \in(0,1)$ such that

$$
\begin{aligned}
& r v_{0} \leq u_{0}<v_{0} \\
& u_{0} \leq A u_{0} \leq A v_{0} \leq v_{0}
\end{aligned}
$$

(ii) operator equation $x=A x$ has a unique solution in $P_{h}$.

Remark 10. An operator $A$ is said to be generalized concave if $A$ satisfies condition $\left(D_{2}\right)$.

\section{Existence and Uniqueness of Positive Solutions for Problem (1)}

In this section, we use Theorem 9 to study problem (1) and we obtain some new results on the existence and uniqueness of positive solutions. This is also the main motivation for the study of (1) in the present work.

Set $J=[0,1], C[J, R]=\{x \mid x: J \rightarrow R$ is continuous $\}$. Evidently, $C[J, R]$ is a Banach space with the norm $\|x\|_{C}=$ $\sup \{|x(t)|: t \in J\}, P=\{x \in C[J, R] \mid x(t) \geq 0, t \in$ $J\}$, the standard cone, and it is normal. Our main result is summarized in the following theorem.

Theorem 11. Assume that $\left(A_{1}\right)-\left(A_{4}\right)$ hold and $f(t, b c+b d)>$ $0, f(t, a d+b d)>0, t \in[0,1]$. Then

(i) there exist $u_{0}, v_{0} \in P_{h}$ such that

$$
\begin{aligned}
& u_{0}(t) \leq \int_{0}^{1} G_{1}(t, \xi) \int_{0}^{1} G_{2}(\xi, s) f\left(s, u_{0}(s)\right) d s d \xi, \\
& t \in J, \\
& v_{0}(t) \geq \int_{0}^{1} G_{1}(t, \xi) \int_{0}^{1} G_{2}(\xi, s) f\left(s, v_{0}(s)\right) d s d \xi,
\end{aligned}
$$


(ii) the integral boundary value problem (1) has a unique positive solution $x^{*}$ in $P_{h}$, where $h(t)=(a t+b)[c(1-$ $t)+d], t \in J$.

Proof. Define an operator $A: C[J, R] \rightarrow C[J, R]$ by

$$
A x(t)=\int_{0}^{1} G_{1}(t, \xi) \int_{0}^{1} G_{2}(\xi, s) f(s, x(s)) d s d \xi .
$$

We know that $x$ is a solution of problem (1) if and only if $x$ is a fixed point of the operator $A$.

Firstly, we show that $A: P \rightarrow P$ is increasing, generalized concave. From $\left(A_{1}\right)$, we know that $f(t, x(t)) \geq 0$, and from (10) and (13) we have $G_{1}(t, s) \geq 0, G_{2}(t, s) \geq 0$, so we have $A x(t) \geq 0$ for $x \in P$. It follows from $\left(A_{1}\right)$ that $A: P \rightarrow P$ is increasing.

Now we prove that $A: P \rightarrow P$ is generalized concave. For any $x \in P$ and $\lambda \in(0,1)$, from $\left(A_{2}\right)$ we have

$$
\begin{aligned}
& A(\lambda x)(t)=\int_{0}^{1} G_{1}(t, \xi) \int_{0}^{1} G_{2}(\xi, s) f(s, \lambda x(s)) d s d \xi \\
& \quad \geq \alpha(\lambda) \int_{0}^{1}(t, \xi) \int_{0}^{1} G_{2}(\xi, s) f(s, x(s)) d s d \xi \\
& =\alpha(\lambda) A x(t) .
\end{aligned}
$$

Thus, we have $A(\lambda x) \geq \alpha(\lambda) A x, x \in P, \lambda \in(0,1)$.

Secondly, we prove that $A h \in P_{h}$. Note that $h(t)=(a t+$ $b)[c(1-t)+d]$, and we have

$$
A h(t)=\int_{0}^{1} G_{1}(t, \xi) \int_{0}^{1} G_{2}(\xi, s) f(s, h(s)) d s d \xi .
$$

From (10), it is easy to obtain that

$$
\begin{aligned}
& \frac{h(t)}{(a+b)(c+d)} G_{1}(\xi, s) \leq G_{1}(t, s) \leq \frac{h(t)}{\rho}, \\
& \forall \xi \in[0,1], t, s \in[0,1] .
\end{aligned}
$$

From (14), we have

$$
m(t) M_{p q} M_{\alpha}(s) \leq G_{2}(t, s) \leq M_{p q} M_{\alpha}(s) .
$$

We know by (16) that $m(t)$ is decreasing, so when $t=1, m(t)$ obtains the minimum, and $m(1)>0$, so we have

$$
m(1) M_{p q} M_{\alpha}(s) \leq G_{2}(t, s) \leq M_{p q} M_{\alpha}(s),
$$

and from (17), we have

$$
\begin{aligned}
\int_{0}^{1} M_{\alpha}(s) d s & =\int_{0}^{1}\left[\frac{2(1-s)^{\alpha-3}}{\Gamma(\alpha-2)}+\frac{(1-s)^{\alpha-4}}{\Gamma(\alpha-3)}\right] d s \\
& =\frac{2}{(\alpha-2) \Gamma(\alpha-2)}+\frac{1}{(\alpha-3) \Gamma(\alpha-3)} .
\end{aligned}
$$

So from (25), (27), and (28), (22) has the following form:

$$
\begin{aligned}
A h(t) & =\int_{0}^{1} G_{1}(t, \xi) \int_{0}^{1} G_{2}(\xi, s) f(s, h(s)) d s d \xi \\
& \leq \frac{h(t)}{\rho} \int_{0}^{1} G_{2}(\xi, s) f(s, h(s)) d s \\
& \leq \frac{h(t)}{\rho} M_{p q} \int_{0}^{1} M_{\alpha}(s) f(s, h(s)) d s .
\end{aligned}
$$

After simple calculation, we get $h^{\prime}\left(t_{0}\right)=0$, where $t_{0}=(a c+$ $a d-b c) / 2 a c$. So we need to discuss several cases:

(i) When $0 \leq t_{0} \leq 1$, we have

$$
f(t, h(t)) \leq f\left(t, h\left(t_{0}\right)\right), \quad t \in[0,1] .
$$

Let $f_{01}=\max \left\{f\left(t, h\left(t_{0}\right)\right), t \in[0,1]\right\}$; then $f_{01} \geq f\left(t, h\left(t_{0}\right)\right) \geq$ $f(t, h(0))=f(t, b c+b d)>0$. From (28) and (29), we obtain that

$$
\begin{aligned}
& A h(t) \leq \frac{h(t)}{\rho} M_{p q} f_{01} \int_{0}^{1} M_{\alpha}(s) d s \\
& =\frac{M_{p q} f_{01}}{\rho}\left[\frac{2}{(\alpha-2) \Gamma(\alpha-2)}+\frac{1}{(\alpha-3) \Gamma(\alpha-3)}\right] \\
& \cdot h(t) .
\end{aligned}
$$

(ii) When $t_{0}>1$, we have

$$
f(t, h(t)) \leq f(t, h(1))=f(t, a d+b d), \quad t \in[0,1] .
$$

Let $f_{02}=\max \{f(t, a d+b d), t \in[0,1]\}$; then $f_{02} \geq f(t$, $h(1))=f(t, a d+b d)>0$. From (28) and (29), we obtain that

$$
\begin{array}{rl}
A & h(t) \leq \frac{h(t)}{\rho} M_{p q} f_{02} \int_{0}^{1} M_{\alpha}(s) d s \\
= & \frac{M_{p q} f_{02}}{\rho}\left[\frac{2}{(\alpha-2) \Gamma(\alpha-2)}+\frac{1}{(\alpha-3) \Gamma(\alpha-3)}\right] \\
\cdot & h(t) .
\end{array}
$$

(iii) When $t_{0}<0$, we have

$$
f(t, h(t)) \leq f(t, h(0))=f(t, b c+b d), \quad t \in[0,1] .
$$

Let $f_{03}=\max \{f(t, b c+b d), t \in[0,1]\}$; then $f_{03} \geq f(t$, $h(0))=f(t, b c+b d)>0$. From (28) and (29), we obtain that

$$
\begin{array}{rl}
A & h(t) \leq \frac{h(t)}{\rho} M_{p q} f_{03} \int_{0}^{1} M_{\alpha}(s) d s \\
= & \frac{M_{p q} f_{03}}{\rho}\left[\frac{2}{(\alpha-2) \Gamma(\alpha-2)}+\frac{1}{(\alpha-3) \Gamma(\alpha-3)}\right] \\
& \cdot h(t) .
\end{array}
$$


Let

$$
\begin{aligned}
\mu_{1}= & \frac{M_{p q}}{\rho}\left[\frac{2}{(\alpha-2) \Gamma(\alpha-2)}+\frac{1}{(\alpha-3) \Gamma(\alpha-3)}\right] \\
& \cdot \begin{cases}f_{01}, & t_{0} \in[0,1] \\
f_{02}, & t_{0}>1 \\
f_{03}, & t_{0}<0 .\end{cases}
\end{aligned}
$$

Then from (31), (33), and (35), we have

$$
A h(t) \leq \mu_{1} h(t), \quad t \in[0,1] .
$$

On the other hand, from (25), (27), and (28), we have

$$
\begin{gathered}
A h(t)=\int_{0}^{1} G_{1}(t, \xi) \int_{0}^{1} G_{2}(\xi, s) f(s, h(s)) d s d \xi \\
\geq \frac{h(t)}{(a+b)(c+d)} \\
\quad \cdot \int_{0}^{1} G_{1}(t, \xi) \int_{0}^{1} G_{2}(\xi, s) f(s, h(s)) d s d \xi \\
\geq \frac{h(t)}{(a+b)(c+d)} m(1) M_{p q} \int_{0}^{1} G_{1}(t, \xi) \\
\cdot \int_{0}^{1} M_{\alpha}(s) f(s, h(s)) d s d \xi .
\end{gathered}
$$

Also, we need to discuss the following cases:

(i) When $0 \leq t_{0} \leq 1$, we have

$$
\begin{aligned}
f(t, h(t)) & \geq \min \{f(t, h(0)), f(t, h(1)), t \in[0,1]\} \\
& :=f_{04} .
\end{aligned}
$$

We can see that $f_{04}=\min \{f(t, h(0)), f(t, h(1)), t \in[0,1]\}>$ 0 . From (28), (38), and Lemma 8 , we obtain that

$$
\begin{aligned}
& A h(t) \geq \frac{h(t)}{(a+b)(c+d)} m(1) \\
& \cdot M_{p q} f_{04}\left[\frac{2}{(\alpha-2) \Gamma(\alpha-2)}+\frac{1}{(\alpha-3) \Gamma(\alpha-3)}\right] \\
& \quad \cdot \int_{0}^{1} G_{1}(\tau, \xi) d \xi \\
& \quad \geq \frac{m(1) M_{p q} f_{04} g_{0}}{(a+b)(c+d)}\left[\frac{2}{(\alpha-2) \Gamma(\alpha-2)}\right. \\
& \left.\quad+\frac{1}{(\alpha-3) \Gamma(\alpha-3)}\right] h(t) .
\end{aligned}
$$

(ii) When $t_{0}>1$, we have

$f(t, h(t)) \geq f(t, h(0))=f(t, b c+b d), \quad t \in[0,1]$.
Let $f_{05}=\min \{f(t, b c+b d), t \in[0,1]\}$, and then $f_{05}>0$. From (28), (38), and Lemma 8, we obtain that

$$
\begin{aligned}
& A h(t) \geq \frac{h(t)}{(a+b)(c+d)} m(1) \\
& \cdot M_{p q} f_{05}\left[\frac{2}{(\alpha-2) \Gamma(\alpha-2)}+\frac{1}{(\alpha-3) \Gamma(\alpha-3)}\right] \\
& \quad \cdot \int_{0}^{1} G_{1}(\tau, \xi) d \xi \\
& \quad \geq \frac{m(1) M_{p q} f_{05} g_{0}}{(a+b)(c+d)}\left[\frac{2}{(\alpha-2) \Gamma(\alpha-2)}\right. \\
& \left.\quad+\frac{1}{(\alpha-3) \Gamma(\alpha-3)}\right] h(t) .
\end{aligned}
$$

(iii) When $t_{0}<0$, we have

$$
f(t, h(t)) \geq f(t, h(1))=f(t, a d+b d), \quad t \in[0,1] .
$$

Let $f_{06}=\min \{f(t, a d+b d), t \in[0,1]\}$, and then $f_{06}>0$. From (28), (38), and Lemma 8, we obtain that

$$
\begin{aligned}
& A h(t) \geq \frac{h(t)}{(a+b)(c+d)} m(1) \\
& \cdot M_{p q} f_{06}\left[\frac{2}{(\alpha-2) \Gamma(\alpha-2)}+\frac{1}{(\alpha-3) \Gamma(\alpha-3)}\right] \\
& \quad \cdot \int_{0}^{1} G_{1}(\tau, \xi) d \xi \\
& \quad \geq \frac{m(1) M_{p q} f_{06} g_{0}}{(a+b)(c+d)}\left[\frac{2}{(\alpha-2) \Gamma(\alpha-2)}\right. \\
& \left.\quad+\frac{1}{(\alpha-3) \Gamma(\alpha-3)}\right] h(t) .
\end{aligned}
$$

Let

$$
\begin{aligned}
\mu_{2}= & \frac{m(1) M_{p q} g_{0}}{(a+b)(c+d)}\left[\frac{2}{(\alpha-2) \Gamma(\alpha-2)}\right. \\
& \left.+\frac{1}{(\alpha-3) \Gamma(\alpha-3)}\right] \begin{cases}f_{04}, & t_{0} \in[0,1], \\
f_{05}, & t_{0}>1, \\
f_{06}, & t_{0}<0 .\end{cases}
\end{aligned}
$$

Then from (40), (42), and (44), we have

$$
A h(t) \geq \mu_{2} h(t), \quad t \in[0,1] .
$$

So, from (37) and (46) we have

$$
\mu_{2} h(t) \leq A h(t) \leq \mu_{1} h(t),
$$


that is, $A h \in P_{h}$. Finally, an application of Theorem 9 implies that

(i) there exist $u_{0}, v_{0} \in P_{h}$ such that $u_{0} \leq A u_{0}, v_{0} \geq A v_{0}$, that is,

$$
\begin{aligned}
& u_{0}(t) \leq \int_{0}^{1} G_{1}(t, \xi) \int_{0}^{1} G_{2}(\xi, s) f\left(s, u_{0}(s)\right) d s d \xi \\
& t \in J, \\
& v_{0}(t) \geq \int_{0}^{1} G_{1}(t, \xi) \int_{0}^{1} G_{2}(\xi, s) f\left(s, v_{0}(s)\right) d s d \xi
\end{aligned}
$$

$t \in J$

(ii) operator equation $x=A x$ has a unique solution $x^{*}$ in $P_{h}$. That is, $x^{*}(t)$ is the unique positive solution for problem (1) in $P_{h}$.

Theorem 12. Assume that $\left(A_{1}^{\prime}\right),\left(A_{2}^{\prime}\right),\left(A_{3}\right),\left(A_{4}\right)$ hold and $f(t, x)>0$ for $x>0, t \in[0,1]$. Then

(i) there exist $u_{0}, v_{0} \in P_{h}$ such that

$$
\begin{aligned}
& u_{0}(t) \leq \int_{0}^{1} G_{1}(t, \xi) \int_{0}^{1} G_{2}(\xi, s) f\left(s, \bar{u}_{0}(s)\right) d s d \xi, \\
& t \in J, \\
& v_{0}(t) \geq \int_{0}^{1} G_{1}(t, \xi) \int_{0}^{1} G_{2}(\xi, s) f\left(s, \bar{v}_{0}(s)\right) d s d \xi
\end{aligned}
$$

where

$$
\begin{aligned}
& \bar{u}_{0}(t)=\int_{0}^{1} G_{1}(t, \xi) \int_{0}^{1} G_{2}(\xi, s) f\left(s, u_{0}(s)\right) d s d \xi \\
& t \in J, \\
& \bar{v}_{0}(t)=\int_{0}^{1} G_{1}(t, \xi) \int_{0}^{1} G_{2}(\xi, s) f\left(s, v_{0}(s)\right) d s d \xi
\end{aligned}
$$

$$
t \in J
$$

(ii) the integral boundary value problem (1) has a unique positive solution $x^{*}$ in $P_{h}$, where $h(t)=(a t+b)[c(1-t)+d], t \in$ J.

Proof. Similar to the proof of Theorem 11, we consider the operator $A: C[J, R] \rightarrow C[J, R]$ by

$$
A x(t)=\int_{0}^{1} G_{1}(t, \xi) \int_{0}^{1} G_{2}(\xi, s) f(s, x(s)) d s d \xi
$$

From $\left(A_{1}^{\prime}\right)$, we know that $A x(t) \geq 0, t \in[0,1]$. It follows from $\left(A_{1}^{\prime}\right)$ that $A: P \rightarrow P$ is decreasing. For any $x \in P, \lambda \in$ $(0,1)$ and from $\left(A_{2}^{\prime}\right)$, we have

$$
\begin{aligned}
& A(\lambda x)(t)=\int_{0}^{1} G_{1}(t, \xi) \int_{0}^{1} G_{2}(\xi, s) f(s, \lambda x(s)) d s d \xi \\
& \quad \leq \lambda^{-\beta(\lambda)} \int_{0}^{1}(t, \xi) \int_{0}^{1} G_{2}(\xi, s) f(s, x(s)) d s d \xi \\
& \quad=\lambda^{-\beta(\lambda)} A x(t) .
\end{aligned}
$$

Thus, we have $A(\lambda x) \leq \lambda^{-\beta(\lambda)} A x(t), x \in P, \lambda \in(0,1)$. Further, for $\lambda \in(0,1), x \in P$, we have

$$
A x=A\left(\lambda \cdot \frac{1}{\lambda} x\right) \leq \lambda^{-\beta(\lambda)} A\left(\frac{1}{\lambda} x\right) .
$$

So we obtain $A((1 / \lambda) x) \geq \lambda^{\beta(\lambda)} A x, x \in P, \lambda \in(0,1)$. Consequently, $A^{2}: P \rightarrow P$ is increasing and for $x \in P, \lambda \in$ $(0,1)$,

$$
\begin{aligned}
A^{2}(\lambda x) & =A(A(\lambda x)) \geq A\left(\lambda^{-\beta(\lambda)} A x\right) \\
& =A\left(\frac{1}{\lambda^{\beta(x)}} A x\right) \geq\left(\lambda^{\beta(\lambda)}\right)^{\beta\left(\lambda^{\beta(\lambda)}\right)} A^{2} x \\
& \geq \lambda^{\beta(\lambda)} A^{2} x .
\end{aligned}
$$

Let $\alpha(t)=t^{\beta(t)}, t \in(0,1)$. Then $t<\alpha(t)<1$ and $A^{2}(\lambda x) \geq$ $\alpha(\lambda) A^{2} x, \lambda \in(0,1), x \in P$. So the operator $A^{2}: P \rightarrow P$ is generalized concave.

Next we prove that $A^{2} h \in P_{h}$. Note that $h(t)=(a t+$ b) $[c(1-t)+d]$, and we can obtain

$$
\begin{aligned}
& A h(t)=\int_{0}^{1} G_{1}(t, \xi) \int_{0}^{1} G_{2}(\xi, s) f(s, h(s)) d s d \xi \\
& A^{2} h(t)=A(A(t)) \\
& \quad=\int_{0}^{1} G_{1}(t, \xi) \int_{0}^{1} G_{2}(\xi, s) f(s, A(h(s))) d s d \xi .
\end{aligned}
$$

Because $h^{\prime}\left(t_{0}\right)=0$, where $t_{0}=(a c+a d-b c) / 2 a c$, we need to discuss several cases:

(i) When $0 \leq t_{0} \leq 1$, we have

$$
\begin{aligned}
f(t, h(t)) & \leq \min \{f(t, h(0)), f(t, h(1)), t \in[0,1]\} \\
& :=f_{04},
\end{aligned}
$$

and we can see that $f_{04}=\min \{f(t, h(0)), f(t, h(1)), t \in$ $[0,1]\}>0$. From (28) and (29), we obtain that

$$
\begin{aligned}
& A h(t) \leq \frac{h(t)}{\rho} M_{p q} f_{04} \int_{0}^{1} M_{\alpha}(s) d s \\
& =\frac{M_{p q} f_{04}}{\rho}\left[\frac{2}{(\alpha-2) \Gamma(\alpha-2)}+\frac{1}{(\alpha-3) \Gamma(\alpha-3)}\right] \\
& \cdot h(t) .
\end{aligned}
$$


(ii) When $t_{0}>1$, we have

$$
f(t, h(t)) \leq f(t, h(0))=f(t, b c+b d), \quad t \in[0,1],
$$

Note that $f_{03}=\max \{f(t, b c+b d), t \in[0,1]\}$; then $f_{03} \geq$ $f(t, h(0))=f(t, b c+b d)>0$. From (28) and (29), we obtain that

$$
\begin{aligned}
A h(t) \leq & \frac{h(t)}{\rho} M_{p q} f_{03} \int_{0}^{1} M_{\alpha}(s) d s \\
= & \frac{M_{p q} f_{03}}{\rho}\left[\frac{2}{(\alpha-2) \Gamma(\alpha-2)}+\frac{1}{(\alpha-3) \Gamma(\alpha-3)}\right] \\
& \cdot h(t)
\end{aligned}
$$

(iii) When $t_{0}<0$, we have

$$
\begin{aligned}
& f(t, h(t)) \leq f(t, h(1))=f(t, a d+b d), \\
& \quad t \in[0,1] .
\end{aligned}
$$

Note that $f_{02}=\max \{f(t, a d+b d), t \in[0,1]\}$; then $f_{02} \geq$ $f(t, h(1))=f(t, a d+b d)>0$. From (28) and (29), we obtain that

$$
\begin{aligned}
& A h(t) \leq \frac{h(t)}{\rho} M_{p q} f_{02} \int_{0}^{1} M_{\alpha}(s) d s \\
& =\frac{M_{p q} f_{02}}{\rho}\left[\frac{2}{(\alpha-2) \Gamma(\alpha-2)}+\frac{1}{(\alpha-3) \Gamma(\alpha-3)}\right] \\
& \quad \cdot h(t) .
\end{aligned}
$$

Let

$$
\begin{aligned}
\mu_{3}= & \frac{M_{p q}}{\rho}\left[\frac{2}{(\alpha-2) \Gamma(\alpha-2)}+\frac{1}{(\alpha-3) \Gamma(\alpha-3)}\right] \\
& \cdot \begin{cases}f_{04}, & t_{0} \in[0,1] \\
f_{03}, & t_{0}>1 \\
f_{02}, & t_{0}<0 .\end{cases}
\end{aligned}
$$

Then from (57), (59), and (61), we have

$$
A h(t) \leq \mu_{3} h(t), \quad t \in[0,1]
$$

Further, we need to discuss the following cases:

(i) When $0 \leq t_{0} \leq 1$, we have

$$
f(t, h(t)) \geq f\left(t, h\left(t_{0}\right)\right), \quad t \in[0,1] .
$$

Let $f_{07}=\min \left\{f\left(t, h\left(t_{0}\right)\right), t \in[0,1]\right\}$, and then $f_{07}>0$. From (28), (38), and Lemma 8, we obtain that

$$
\begin{aligned}
& A h(t) \geq \frac{h(t)}{(a+b)(c+d)} m(1) \\
& \cdot M_{p q} f_{07}\left[\frac{2}{(\alpha-2) \Gamma(\alpha-2)}+\frac{1}{(\alpha-3) \Gamma(\alpha-3)}\right] \\
& \quad \cdot \int_{0}^{1} G_{1}(\tau, \xi) d \xi \\
& \quad \geq \frac{m(1) M_{p q} f_{07} g_{0}}{(a+b)(c+d)}\left[\frac{2}{(\alpha-2) \Gamma(\alpha-2)}\right. \\
& \left.\quad+\frac{1}{(\alpha-3) \Gamma(\alpha-3)}\right] h(t) .
\end{aligned}
$$

(ii) When $t_{0}>1$, we have

$$
f(t, h(t)) \geq f(t, h(1))=f(t, a d+b d),
$$

$$
t \in[0,1] .
$$

Note that $f_{06}=\min \{f(t, a d+b d), t \in[0,1]\}>0$ and from (28), (38), and Lemma 8, we obtain that

$$
\begin{aligned}
& A h(t) \geq \frac{h(t)}{(a+b)(c+d)} m(1) \\
& \cdot M_{p q} f_{06}\left[\frac{2}{(\alpha-2) \Gamma(\alpha-2)}+\frac{1}{(\alpha-3) \Gamma(\alpha-3)}\right] \\
& \quad \cdot \int_{0}^{1} G_{1}(\tau, \xi) d \xi \\
& \quad \geq \frac{m(1) M_{p q} f_{06} g_{0}}{(a+b)(c+d)}\left[\frac{2}{(\alpha-2) \Gamma(\alpha-2)}\right. \\
& \left.\quad+\frac{1}{(\alpha-3) \Gamma(\alpha-3)}\right] h(t) .
\end{aligned}
$$

(iii) When $t_{0}<0$, we have

$f(t, h(t)) \geq f(t, h(0))=f(t, b c+b d), \quad t \in[0,1]$.

Note that $f_{05}=\min \{f(t, b c+b d), t \in[0,1]\}>0$ and from (28), (38), and Lemma 8, we obtain that

$$
\begin{aligned}
& A h(t) \geq \frac{h(t)}{(a+b)(c+d)} m(1) \\
& \cdot M_{p q} f_{05}\left[\frac{2}{(\alpha-2) \Gamma(\alpha-2)}+\frac{1}{(\alpha-3) \Gamma(\alpha-3)}\right] \\
& \quad \cdot \int_{0}^{1} G_{1}(\tau, \xi) d \xi \\
& \quad \geq \frac{m(1) M_{p q} f_{05} g_{0}}{(a+b)(c+d)}\left[\frac{2}{(\alpha-2) \Gamma(\alpha-2)}\right. \\
& \left.\quad+\frac{1}{(\alpha-3) \Gamma(\alpha-3)}\right] h(t) .
\end{aligned}
$$


Let

$$
\begin{aligned}
\mu_{4} & =\frac{m(1) M_{p q} g_{0}}{(a+b)(c+d)}\left[\frac{2}{(\alpha-2) \Gamma(\alpha-2)}\right. \\
& \left.+\frac{1}{(\alpha-3) \Gamma(\alpha-3)}\right] \begin{cases}f_{07}, & t_{0} \in[0,1], \\
f_{06}, & t_{0}>1, \\
f_{05}, & t_{0}<0 .\end{cases}
\end{aligned}
$$

Then from (65), (67), and (69), we have

$$
A h(t) \geq \mu_{4} h(t), \quad t \in[0,1] .
$$

So, from (63) and (71) we have

$$
\mu_{4} h(t) \leq A h(t) \leq \mu_{3} h(t) .
$$

Hence, we have

$$
\begin{aligned}
& A^{2} h(t) \leq \int_{0}^{1} G_{1}(t, \xi) \int_{0}^{1} G_{2}(\xi, s) f\left(s, \mu_{4} h(s)\right) d s d \xi \\
& A^{2} h(t) \geq \int_{0}^{1} G_{1}(t, \xi) \int_{0}^{1} G_{2}(\xi, s) f\left(s, \mu_{3} h(s)\right) d s d \xi
\end{aligned}
$$

Because $h^{\prime}\left(t_{0}\right)=0$, where $t_{0}=(a c+a d-b c) / 2 a c$, we need to discuss the following cases:

(i) When $0 \leq t_{0} \leq 1$, we have

$$
\begin{aligned}
f & \left(t, \mu_{4} h(t)\right) \\
& \leq \min \left\{f\left(t, \mu_{4} h(0)\right), f\left(t, \mu_{4} h(1)\right), t \in[0,1]\right\} \\
& :=f_{08},
\end{aligned}
$$

and we can see that $f_{08}=\min \left\{f\left(t, \mu_{4} h(0)\right), f\left(t, \mu_{4} h(1)\right), t \in\right.$ $[0,1]\}>0$. From (28) and (73), we obtain that

$$
\begin{aligned}
A^{2} h(t) & \leq \frac{h(t)}{\rho} M_{p q} f_{08} \int_{0}^{1} M_{\alpha}(s) d s \\
\quad= & \frac{M_{p q} f_{08}}{\rho}\left[\frac{2}{(\alpha-2) \Gamma(\alpha-2)}+\frac{1}{(\alpha-3) \Gamma(\alpha-3)}\right]
\end{aligned}
$$$$
\cdot h(t) \text {. }
$$

(ii) When $t_{0}>1$, we have

$$
\begin{array}{r}
f\left(t, \mu_{4} h(t)\right) \leq f\left(t, \mu_{4} h(0)\right)=f\left(t, \mu_{4}(b c+b d)\right), \\
t \in[0,1] .
\end{array}
$$

Let $f_{09}=\max \left\{f\left(t, \mu_{4}(b c+b d)\right), t \in[0,1]\right\}$; then $f_{09} \geq$ $f\left(t, \mu_{4} h(0)\right)=f\left(t, \mu_{4}(b c+b d)\right)>0$. From (28) and (73), we obtain that

$$
\begin{aligned}
A^{2} h(t) & \leq \frac{h(t)}{\rho} M_{p q} f_{09} \int_{0}^{1} M_{\alpha}(s) d s \\
\quad & \frac{M_{p q} f_{09}}{\rho}\left[\frac{2}{(\alpha-2) \Gamma(\alpha-2)}+\frac{1}{(\alpha-3) \Gamma(\alpha-3)}\right] \\
& \cdot h(t) .
\end{aligned}
$$

(iii) When $t_{0}<0$, we have

$$
\begin{array}{r}
f\left(t, \mu_{4} h(t)\right) \leq f\left(t, \mu_{4} h(1)\right)=f\left(t, \mu_{4}(a d+b d)\right), \\
t \in[0,1] .
\end{array}
$$

Let $f_{10}=\max \left\{f\left(t, \mu_{4}(a d+b d)\right), t \in[0,1]\right\}$; then $f_{10} \geq$ $f\left(t, \mu_{4} h(1)\right)=f\left(t, \mu_{4}(a d+b d)\right)>0$. From (28) and (73), we obtain that

$$
\begin{aligned}
A^{2} h(t) & \leq \frac{h(t)}{\rho} M_{p q} f_{10} \int_{0}^{1} M_{\alpha}(s) d s \\
\quad & \frac{M_{p q} f_{10}}{\rho}\left[\frac{2}{(\alpha-2) \Gamma(\alpha-2)}+\frac{1}{(\alpha-3) \Gamma(\alpha-3)}\right] \\
\cdot h(t) . &
\end{aligned}
$$

Let

$$
\begin{aligned}
\mu_{1}^{\prime}= & \frac{M_{p q}}{\rho}\left[\frac{2}{(\alpha-2) \Gamma(\alpha-2)}+\frac{1}{(\alpha-3) \Gamma(\alpha-3)}\right] \\
& \cdot \begin{cases}f_{08}, & t_{0} \in[0,1], \\
f_{09}, & t_{0}>1, \\
f_{10}, & t_{0}<0 .\end{cases}
\end{aligned}
$$

Then from (76), (78), and (80), we have

$$
A^{2} h(t) \leq \mu_{1}^{\prime} h(t), \quad t \in[0,1] .
$$

Similarly, we need to discuss the following cases:

(i) When $0 \leq t_{0} \leq 1$, we have

$$
f\left(t, \mu_{3} h(t)\right) \geq f\left(t, \mu_{3} h\left(t_{0}\right)\right), \quad t \in[0,1] .
$$

Let $f_{11}=\min \left\{f\left(t, \mu_{3} h\left(t_{0}\right)\right), t \in[0,1]\right\}$, and then $f_{11}>0$. From (28), (74), and Lemma 8, we obtain that

$$
\begin{aligned}
& A^{2} h(t) \geq \frac{h(t)}{(a+b)(c+d)} m(1) \\
& \cdot M_{p q} f_{11}\left[\frac{2}{(\alpha-2) \Gamma(\alpha-2)}+\frac{1}{(\alpha-3) \Gamma(\alpha-3)}\right] \\
& \quad \cdot \int_{0}^{1} G_{1}(\tau, \xi) d \xi \\
& \quad \geq \frac{m(1) M_{p q} f_{11} g_{0}}{(a+b)(c+d)}\left[\frac{2}{(\alpha-2) \Gamma(\alpha-2)}\right. \\
& \left.\quad+\frac{1}{(\alpha-3) \Gamma(\alpha-3)}\right] h(t) .
\end{aligned}
$$

(ii) When $t_{0}>1$, we have

$$
\begin{array}{r}
f\left(t, \mu_{3} h(t)\right) \geq f\left(t, \mu_{3} h(1)\right)=f\left(t, \mu_{3}(a d+b d)\right), \\
t \in[0,1] .
\end{array}
$$


Let $f_{12}=\min \left\{f\left(t, \mu_{3}(a d+b d)\right), t \in[0,1]\right\}$, and then $f_{12}>0$. From (28), (74), and Lemma 8, we obtain that

$$
\begin{aligned}
& A^{2} h(t) \geq \frac{h(t)}{(a+b)(c+d)} m(1) \\
& \cdot M_{p q} f_{12}\left[\frac{2}{(\alpha-2) \Gamma(\alpha-2)}+\frac{1}{(\alpha-3) \Gamma(\alpha-3)}\right] \\
& \cdot \int_{0}^{1} G_{1}(\tau, \xi) d \xi \\
& \quad \geq \frac{m(1) M_{p q} f_{12} g_{0}}{(a+b)(c+d)}\left[\frac{2}{(\alpha-2) \Gamma(\alpha-2)}\right. \\
& \left.\quad+\frac{1}{(\alpha-3) \Gamma(\alpha-3)}\right] h(t) .
\end{aligned}
$$

(iii) When $t_{0}<0$, we have

$$
\begin{array}{r}
f\left(t, \mu_{3} h(t)\right) \geq f\left(t, \mu_{3} h(0)\right)=f\left(t, \mu_{3}(b c+b d)\right), \\
t \in[0,1] .
\end{array}
$$

Note that $f_{13}=\min \left\{f\left(t, \mu_{3}(b c+b d)\right), t \in[0,1]\right\}>0$ and from (28), (74), and Lemma 8, we obtain that

$$
\begin{aligned}
& A^{2} h(t) \geq \frac{h(t)}{(a+b)(c+d)} m(1) \\
& \cdot M_{p q} f_{13}\left[\frac{2}{(\alpha-2) \Gamma(\alpha-2)}+\frac{1}{(\alpha-3) \Gamma(\alpha-3)}\right] \\
& \quad \cdot \int_{0}^{1} G_{1}(\tau, \xi) d \xi \\
& \quad \geq \frac{m(1) M_{p q} f_{13} g_{0}}{(a+b)(c+d)}\left[\frac{2}{(\alpha-2) \Gamma(\alpha-2)}\right. \\
& \left.\quad+\frac{1}{(\alpha-3) \Gamma(\alpha-3)}\right] h(t) .
\end{aligned}
$$

Let

$$
\begin{aligned}
\mu_{2}^{\prime} & =\frac{m(1) M_{p q} g_{0}}{(a+b)(c+d)}\left[\frac{2}{(\alpha-2) \Gamma(\alpha-2)}\right. \\
& \left.+\frac{1}{(\alpha-3) \Gamma(\alpha-3)}\right] \begin{cases}f_{11}, & t_{0} \in[0,1], \\
f_{12}, & t_{0}>1, \\
f_{13}, & t_{0}<0 .\end{cases}
\end{aligned}
$$

Then from (84), (86), and (88), we have

$$
A^{2} h(t) \geq \mu_{2}^{\prime} h(t), \quad t \in[0,1] .
$$

From (82) and (90), we can get

$$
\mu_{2}^{\prime} h(t) \leq A^{2} h(t) \leq \mu_{1}^{\prime} h(t),
$$

that is, $A^{2} h \in P_{h}$. Finally, an application of Theorem 9 implies the following: (i) there exist $u_{0}, v_{0} \in P_{h}$ such that $u_{0} \leq$
$A^{2} u_{0}, v_{0} \geq A^{2} v_{0}$; (ii) $A^{2} x=x$ has a unique solution $x^{*}$ in $P_{h}$. Let $\bar{u}_{0}=A u_{0}, \bar{v}_{0}=A v_{0}$, and then $u_{0} \leq A \bar{u}_{0}, v_{0} \geq A \bar{v}_{0}$. That is,

$$
\begin{array}{r}
u_{0}(t) \leq \int_{0}^{1} G_{1}(t, \xi) \int_{0}^{1} G_{2}(\xi, s) f\left(s, \bar{u}_{0}(s)\right) d s d \xi, \\
t \in J, \\
v_{0}(t) \geq \int_{0}^{1} G_{1}(t, \xi) \int_{0}^{1} G_{2}(\xi, s) f\left(s, \bar{v}_{0}(s)\right) d s d \xi, \\
t \in J .
\end{array}
$$

Moreover, $A^{2} x^{*}=x^{*}$. Next, we show that $x^{*}$ is the unique fixed point of $A$ in $P_{h}$. In view of $A^{2}\left(A x^{*}\right)=A\left(A^{2} x^{*}\right)=A x^{*}$, by the uniqueness of solutions for the operator equation $x=$ $A^{2} x$, we have that $A x^{*}=x^{*}$. Suppose that $y$ is another fixed point of $A$ in $P_{h}$, so we have $A^{2} y=A(A y)=A y=y$. Hence, we have $x^{*}=y$. So problem (1) has a unique positive solution $x^{*}$ in $P_{h}$.

\section{Examples}

Example 1. Consider the following fractional boundary value problem of form (1):

$$
\begin{aligned}
& { }^{C} D_{0^{+}}^{11 / 3} x(t)+(1-t)^{1 / 3} x^{1 / 3}+1=0, \quad t \in(0,1), \\
& x(0)=x(1)=0 \\
& x^{\prime \prime}(0)+x^{\prime \prime \prime}(0)=\int_{0}^{1} x^{\prime \prime}(\tau) d\left(\frac{1}{2} \tau\right), \\
& x^{\prime \prime}(1)+x^{\prime \prime \prime}(1)+\int_{0}^{1} x^{\prime \prime}(\tau) d\left(\frac{1}{6} \tau\right)=0 .
\end{aligned}
$$

Conclusion 1. Problem (93) has a unique positive solution in $P_{h}$, where $h(t)=t(1-t), t \in[0,1]$.

Proof. From (93), we know $\alpha=11 / 3, f(t, x)=(1-t)^{1 / 3} x^{1 / 3}+$ 1 , and it is not difficult to find the following:

(1) $f(t, b c+b d)=f(t, a d+b d)=f(t, 0)=1>$ $0, t \in[0,1]$ and $f(t, x)$ is continuous, increasing in $x \in[0,+\infty)$.

(2) Let $\alpha(\lambda)=\lambda^{1 / 3}$, then $\alpha(\lambda) \in(\lambda, 1)$, and we have

$$
\begin{aligned}
f(t, \lambda x) & =(1-t)^{1 / 3}(\lambda x)^{1 / 3}+1 \\
& \geq \lambda^{1 / 3}\left[(1-t)^{1 / 3} x^{1 / 3}+1\right]=\alpha(\lambda) f(t, x) .
\end{aligned}
$$


(3) From (93), we know

$$
\begin{aligned}
p(t) & =\frac{1}{2} t, \\
q(t) & =\frac{1}{6} t, \\
\int_{0}^{1} d p(t) & =\frac{1}{2}, \\
\int_{0}^{1} d q(t) & =\frac{1}{6},
\end{aligned}
$$

so we can get

$$
\begin{aligned}
& \frac{2+\int_{0}^{1} \tau d q(\tau)}{1+\int_{0}^{1} d q(\tau)}=\frac{2+\int_{0}^{1} \tau d((1 / 6) \tau)}{1+\int_{0}^{1} d((1 / 6) \tau)}=\frac{25}{14} \\
& \frac{1-\int_{0}^{1} \tau d p(\tau)}{1-\int_{0}^{1} d p(\tau)}=\frac{1-\int_{0}^{1} \tau d((1 / 2) \tau)}{1-\int_{0}^{1} d((1 / 2) \tau)}=\frac{21}{14}
\end{aligned}
$$

(4)

$$
\begin{gathered}
\int_{0}^{1}(1-s)^{11 / 3-4}\left[(1-s)^{1 / 3} x^{1 / 3}(s)+1\right] d s \\
=\int_{0}^{1} x^{1 / 3}(s) d s+\int_{0}^{1}(1-s)^{-1 / 3} d s
\end{gathered}
$$

exists.

So we claim that the conditions $\left(A_{1}\right)-\left(A_{4}\right)$ hold. Hence, by Theorem 11, we can obtain that (93) has a unique positive solution in $P_{h}$, where $h(t)=t(1-t), t \in[0,1]$.

Example 2. Consider the following fractional boundary value problem of form (1):

$$
\begin{aligned}
& { }^{C} D_{0^{+}}^{11 / 3} x(t)+\frac{(1-t)^{1 / 3}}{1+x^{1 / 3}}+1=0, \quad t \in(0,1), \\
& x(0)=x(1)=0, \\
& x^{\prime \prime}(0)+x^{\prime \prime \prime}(0)=\int_{0}^{1} x^{\prime \prime}(\tau) d\left(\frac{1}{2} \tau\right), \\
& x^{\prime \prime}(1)+x^{\prime \prime \prime}(1)+\int_{0}^{1} x^{\prime \prime}(\tau) d\left(\frac{1}{6} \tau\right)=0 .
\end{aligned}
$$

Conclusion 2. Problem (98) has a unique positive solution in $P_{h}$, where $h(t)=t(1-t), t \in[0,1]$.

Proof. From (98), we know $\alpha=11 / 3, f(t, x)=(1-t)^{1 / 3} /(1+$ $\left.x^{1 / 3}\right)+1$, and it is not difficult to find the following:

(1) $f(t, x) \geq 1>0, t \in[0,1], x \in[0,+\infty)$ and $f(t, x)$ is decreasing in $x \in[0,+\infty)$.

(2) Let $\beta(\lambda)=1 / 3$, and then $\beta(\lambda) \in(0,1)$, so we have

$$
\begin{aligned}
f(t, \lambda x) & =\frac{(1-t)^{1 / 3}}{1+(\lambda x)^{1 / 3}}+1 \leq \lambda^{-1 / 3}\left[\frac{(1-t)^{1 / 3}}{1+x^{1 / 3}}+1\right] \\
& =\lambda^{-\beta(\lambda)} f(t, x) .
\end{aligned}
$$

(3) From (98), we know

$$
\begin{aligned}
p(t) & =\frac{1}{2} t, \\
q(t) & =\frac{1}{6} t, \\
\int_{0}^{1} d p(t) & =\frac{1}{2}, \\
\int_{0}^{1} d q(t) & =\frac{1}{6},
\end{aligned}
$$

so we can get

$$
\begin{aligned}
& \frac{2+\int_{0}^{1} \tau d q(\tau)}{1+\int_{0}^{1} d q(\tau)}=\frac{2+\int_{0}^{1} \tau d((1 / 6) \tau)}{1+\int_{0}^{1} d((1 / 6) \tau)}=\frac{25}{14} \\
& \frac{1-\int_{0}^{1} \tau d p(\tau)}{1-\int_{0}^{1} d p(\tau)}=\frac{1-\int_{0}^{1} \tau d((1 / 2) \tau)}{1-\int_{0}^{1} d((1 / 2) \tau)}=\frac{21}{14}
\end{aligned}
$$

(4)

$$
\begin{aligned}
& \int_{0}^{1}(1-s)^{11 / 3-4}\left[\frac{(1-s)^{1 / 3}}{1+x^{1 / 3}(s)}+1\right] d s \\
& \quad=\int_{0}^{1} \frac{1}{1+x^{1 / 3}(s)} d s+\int_{0}^{1}(1-s)^{-1 / 3} d s
\end{aligned}
$$

exists.

So the conditions $\left(A_{1}^{\prime}\right),\left(A_{2}^{\prime}\right),\left(A_{3}\right),\left(A_{4}\right)$ hold. Hence, by Theorem 12 , we can obtain that $(98)$ has a unique positive solution in $P_{h}$, where $h(t)=t(1-t), t \in[0,1]$.

\section{Conflicts of Interest}

The authors declare that they have no conflicts of interest regarding the publication of this paper.

\section{Authors' Contributions}

The authors declare that they share equal responsibility. All authors read and approved the final manuscript.

\section{Acknowledgments}

This paper was supported financially by the Youth Science Foundation of China (11201272), Shanxi Province Science Foundation (2015011005), and the Youth Science Foundation of Shanxi University of Finance and Economic (2014026).

\section{References}

[1] R. P. Agarwal, D. O’Regan, and S. Staněk, "Positive solutions for Dirichlet problems of singular nonlinear fractional differential equations," Journal of Mathematical Analysis and Applications, vol. 371, no. 1, pp. 57-68, 2010. 
[2] B. Ahmad and J. J. Nieto, "Riemann-Liouville fractional integrodifferential equations with fractional nonlocal integral boundary conditions," Boundary Value Problems, vol. 2011, article 36, 2011.

[3] E. Bazhlekova, Fractional Evolution Equations in Banach Spaces, Eindhoven University of Technology, 2001.

[4] Y. Chang and J. J. Nieto, "Some new existence results for fractional differential inclusions with boundary conditions," Mathematical and Computer Modelling, vol. 49, pp. 605-609, 2009.

[5] B. De Andrade and J. P. C. Dos Santos, "Existence of solutions for a fractional neutral integro-differential equation with unbounded delay," Electronic Journal of Differential Equations, vol. 90, pp. 1-13, 2012.

[6] J. V. Devi and V. Lakshmikantham, "Nonsmooth analysis and fractional differential equations," Nonlinear Analysis, vol. 70, pp. 4151-4157, 2009.

[7] Z. Han, H. Lu, and C. Zhang, "Positive solutions for eigenvalue problems of fractional differential equation with generalized $p$ Laplacian," Applied Mathematics and Computation, vol. 257, pp. 526-536, 2015.

[8] J. Klafter, S. C. Lim, and R. Metzler, Fractional Dynamics, Recent Advances, World Scientific, Singapore, 2011.

[9] A. A. Kilbas, H. M. Srivastava, and J. J. Trujillo, Theory and Applications of Fractional Differential Equations, Elsevier, Amaterdam, 2006.

[10] V. Lakshmikantham and S. Leela, "A Krasnoselskii-Krein-typy uniqueness result for fractional differential equations," Nonlinear Analysis, vol. 71, pp. 3421-3424, 2009.

[11] S. Liang and J. Zhang, "Positive solutions for boundary value problems of nonlinear fractional differential equation," Nonlinear Analysis, vol. 71, pp. 5545-5550, 2009.

[12] I. Podlubny, Fractional Differential Equations, Academic Press, San Diego, Calif, USA, 1999.

[13] W. Wang and X. Guo, "Eigenvalue problem for fractional differential equations with nonlinear integral and disturbance parameter in boundary conditions," Boundary Value Problems, vol. 2016, Article ID 42, 2016.

[14] C. Zhai and L. Xu, "Properties of positive solutions to a class of four-point boundary value problem of Caputo fractional differential equations with a parameter," Communications in Nonlinear Science and Numerical Simulation, vol. 19, no. 8, pp. 2820-2827, 2014.

[15] R. Sakthivel, P. Revathi, and Y. Ren, "Existence of solutions for nonlinear fractional stochastic differential equations," Nonlinear Analysis: Theory, Methods \& Applications, vol. 81, pp. 70-86, 2013.

[16] Z. Wei, W. Dong, and J. Che, "Periodic boundary value problems for fractional differential equations involving a RiemannLiouville fractional derivative," Nonlinear Analysis: Theory, Methods \& Applications, vol. 73, pp. 3232-3238, 2010.

[17] J. Xu, Z. Wei, and W. Dong, "Uniqueness of positive solutions for a class of fractional boundary value problems," Applied Mathematics Letters, vol. 25, no. 3, pp. 590-593, 2012.

[18] Z. Wei, Q. Li, and J. Che, "Initial value problems for fractional differential equations involving Riemann-Liouville sequential fractional derivative," Journal of Mathematical Analysis and Applications, vol. 367, no. 1, pp. 260-272, 2010.

[19] Z. Wei, C. Pang, and Y. Ding, "Positive solutions of singular Caputo fractional differential equations with integral boundary conditions," Communications in Nonlinear Science and Numerical Simulation, vol. 17, no. 8, pp. 3148-3160, 2012.
[20] C. Yang and C. Zhai, "Uniqueness of positive solutions for a fractional differential equation via a fixed point theorem of a sum operator," Electronic Journal of Differential Equations, vol. 70, pp. 1-8, 2012.

[21] Z. Yang, "Existence of nontrivial solutions for a nonlinear Sturm-Liouville problem with integral boundary conditions," Nonlinear Analysis: Theory, Methods \& Applications, vol. 68, pp. 216-225, 2008.

[22] Z. Yang, "Existence and nonexistence results for positive solutions of an integral boundary value problem," Nonlinear Analysis: Theory, Methods \& Applications, vol. 65, pp. 1489-1511, 2006.

[23] C. Zhai, W. Yan, and C. Yang, "A sum operator method for the existence and uniqueness of positive solutions to RiemannLiouville fractional differential equation boundary value problems," Communications in Nonlinear Science and Numerical Simulation, vol. 18, no. 4, pp. 858-866, 2013.

[24] C. Zhai and J. Ren, "Positive and negative solutions of a boundary value problem for a fractional q-difference equation," Advances in Difference Equations, vol. 2017, Article ID 82, 2017.

[25] S. Zhang, "Positive solutions for boundary-value problems of nonlinear fractional differential equations," Electronic Journal of Differential Equations, vol. 36, pp. 1-12, 2006.

[26] H. Feng and C. Zhai, "Existence and uniqueness of positive solutions for a class of fractional differential equation with integral boundary conditions," Nonlinear Analysis: Modelling and Control, vol. 22, no. 2, pp. 160-172, 2017.

[27] C.-B. Zhai, C. Yang, and X.-Q. Zhang, "Positive solutions for nonlinear operator equations and several classes of applications," Mathematische Zeitschrift, vol. 266, no. 1, pp. 43-63, 2010. 


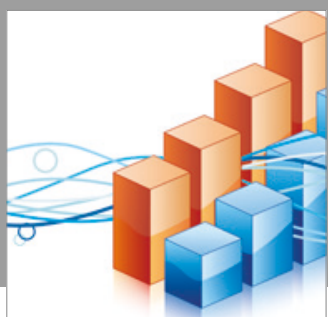

Advances in

Operations Research

vatersals

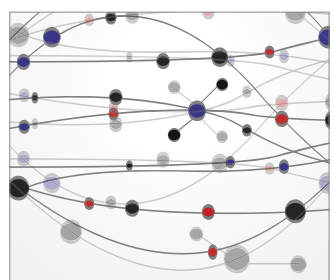

\section{The Scientific} World Journal
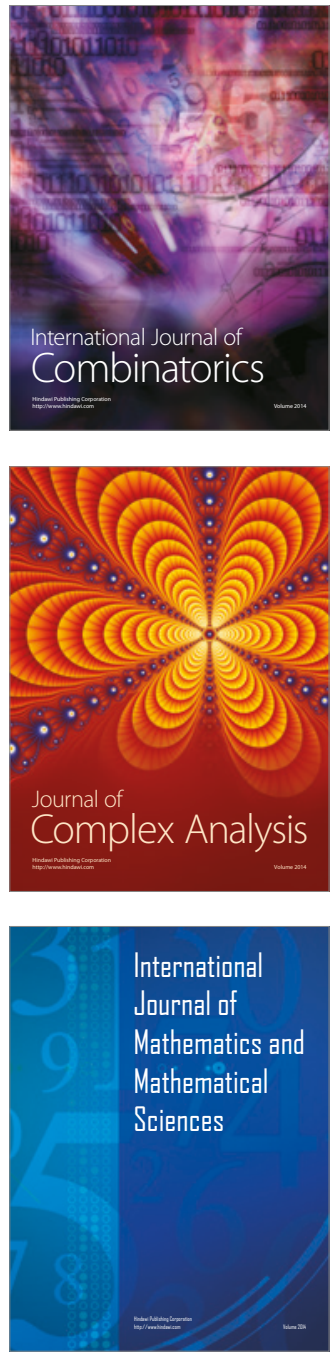
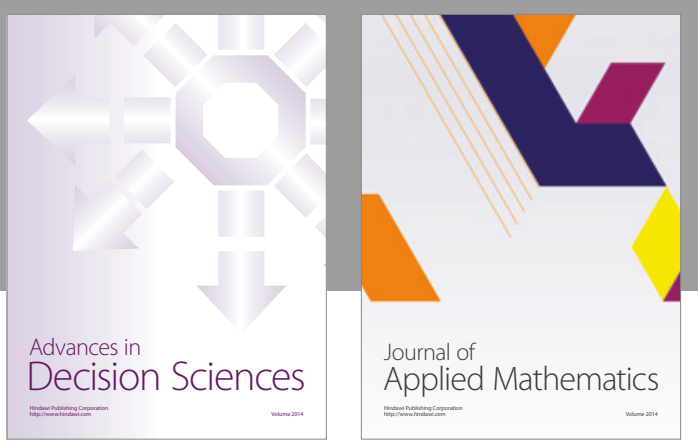

Algebra

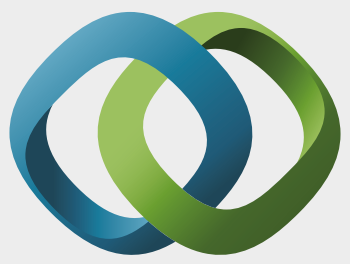

\section{Hindawi}

Submit your manuscripts at

https://www.hindawi.com
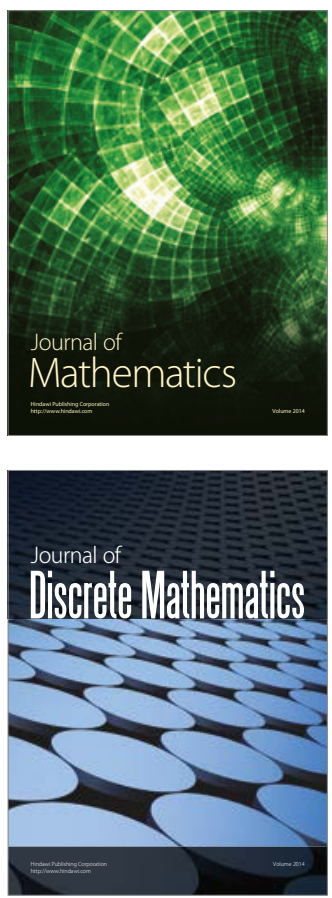

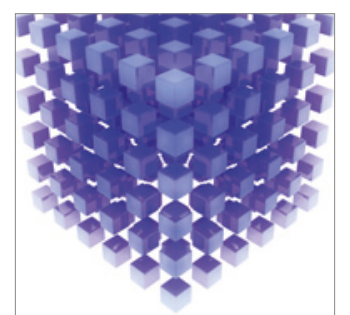

Mathematical Problems in Engineering
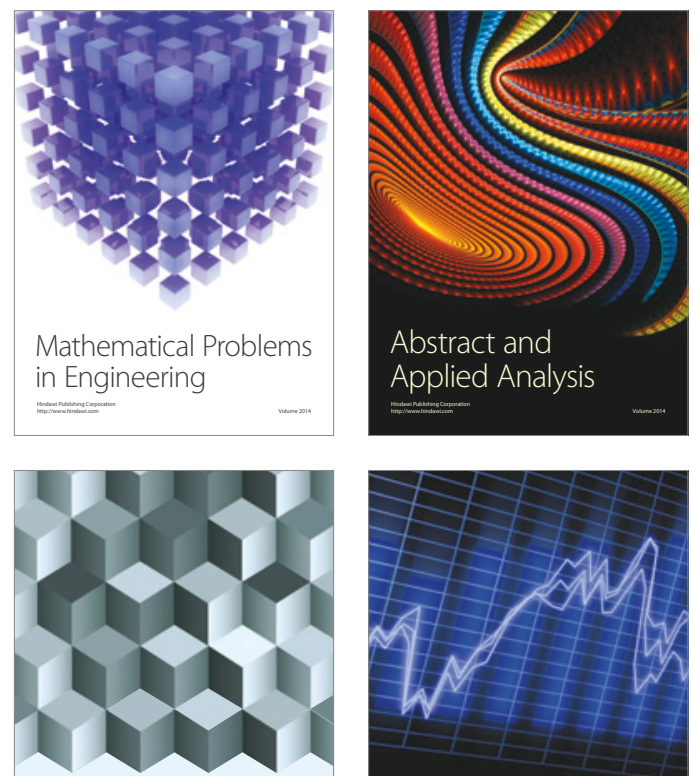

Journal of

Function Spaces

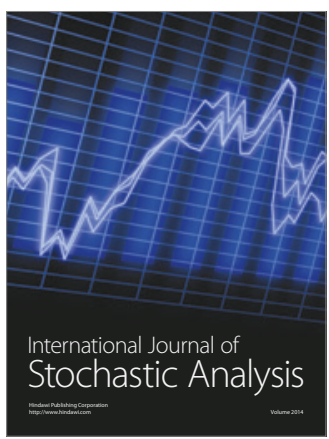

Probability and Statistics
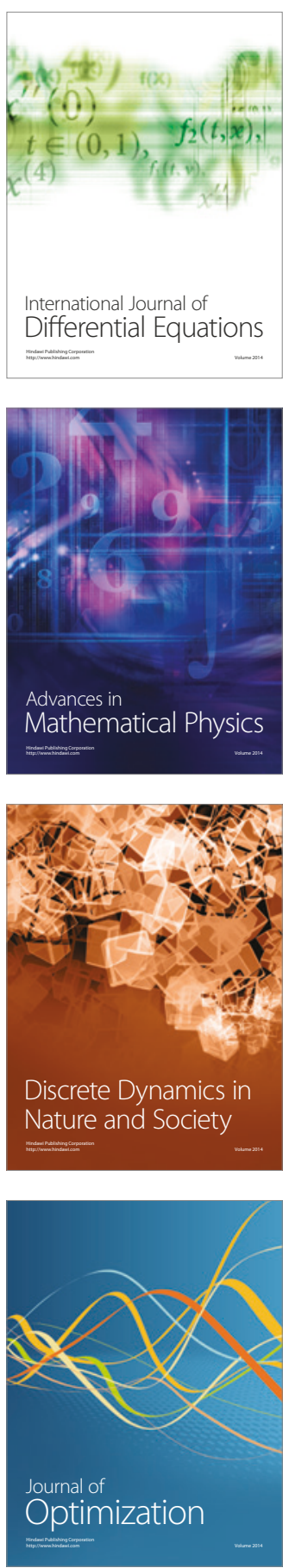